\title{
ON THE STRUCTURE OF ABELIAN $p$-GROUPS
}

BY

\author{
PAUL HILL ${ }^{1}$
}

\begin{abstract}
A new kind of abelian $p$-group, called an $A$-group, is introduced. This class contains the totally projective groups and Warfield's $S$-groups as special cases. It also contains the $N$-groups recently classified by the author. These more general groups are classified by cardinal (numerical) invariants which include, but are not limited to, the Ulm-Kaplansky invariants. Thus the existing theory, as well as the classification, of certain abelian $p$-groups is once again generalized.

Having classified $A$-groups (by means of a uniqueness and corresponding existence theorem) we can successfully study their structure and special properties. Such a study is initiated in the last section of the paper.
\end{abstract}

1. Introduction. In this paper we determine the structure of a class of abelian $p$-groups more general than totally projective groups, $S$-groups, and $N$-groups, all of which have been classified earlier $[5,18,6]$. To introduce this new class of groups, we begin with the following definition. It is to be understood throughout that all groups are abelian.

Definition. If $\mu$ is a limit ordinal, the class $A_{\mu}$ consists of those $p$-groups $H$ for which there is a containing totally projective $p$-group $G$ of length not exceeding $\mu$ that satisfies the following conditions.

(a) $H$ is isotype in $G$.

(b) $p^{\lambda}(G / H)=\left\langle p^{\lambda} G, H\right\rangle / H$ whenever $\lambda<\mu$.

(c) $G / H$ is the direct sum of a totally projective group and a divisible group.

The members of the class $A_{\mu}$ are called $\mu$-elementary $A$-groups. An $A$-group is a direct sum of $\mu$-elementary $A$-groups for various limit ordinals $\mu$. If $G$ is a totally projective $p$-group of length $\mu$, the pair $(H, G)$ is called an $A_{\mu}$-pair whenever conditions (a)-(c) are satisfied. Thus, if $H$ is an $A$-group, then $H=\sum \oplus H_{i}$, where $\left(H_{i}, G_{i}\right)$ is an $A_{\mu(i)}$-pair for distinct limit limit ordinals $\mu(i)$.

Incidentally, if the terminology needs an explanation (and we hope not), one can interpret the letter " $A$ " used here as an abbreviation for the word "accessible", which will prove to be a rather descriptive term. Certainly we follow tradition in using a single letter to represent this class of groups since their predecessors include $T$-groups, $S$-groups, and $N$-groups.

Received by the editors February 8, 1983 and, in revised form, December 15, 1983.

1980 Mathematics Subject Classification. Primary 20K27.

Key words and phrases. Totally projective group, $S$-groups, isotype subgroup, extending isomorphisms, invariants, uniqueness and existence theorems, classification of abelian $p$-groups.

${ }^{1}$ This research was sponsored by NSF Grant MCS 8102470 . 
We are able to show that the structure of an $A$-group is completely determined by certain cardinal invariants including, but not limited to, the Ulm-Kaplansky invariants. The complete collection of invariants are called $A$-invariants (primarily because they suffice for $A$-groups). Observe that if we choose $H=G$ to form the $A_{\mu}$-pair $(H, G)$, we demonstrate that any totally projective group $G$ is an $A$-group. Moreover, it follows from [18] that any $S$-group is an $A$-group and corresponds to the special case where $G_{i} / H_{i}$ is divisible, for each $i$, in the representation $H=$ $\sum \oplus H_{i}$, where $\left(H_{i}, G_{i}\right)$ is an $A_{\mu(i)}$-pair (whereas $G_{i} / H_{i}=0$ corresponds to a totally projective). But the new class also contains groups that are not $S$-groups. In particular, the $N$-group constructed by Megibben in [11] (and referred to by Warfield [18, p. 166] as an example of an isotype subgroup of a totally projective group that is not an $S$-group) is an $A$-group. A general existence theorem proved herein demonstrates the existence of many other $A$-groups.

Warfield's Problem 4 in [18] is the following. Is there a reasonable structure theory for a larger class of groups which are isotype subgroups of totally projective groups? The results of this paper establish a clear answer in the affirmative. Incidentally, Warfield once indicated that he thought the answer to this question was "no". Although this paper generalizes the main results of [18], our approach is somewhat different from that of Warfield. In particular, we do not require cotorsion completions. Otherwise, the techniques employed here in generalizing the theory of $S$-groups to a larger class of $p$-groups are similar to those of [18]. In fact, both are based on my original classification paper [5].

The reader who is familiar with Warfield's paper [18] knows the historical development of the classification of abelian p-groups, at least up through the classification of $S$-groups. Major steps in this development include: (1) countable groups (Ulm, 1933 [16]), (2) direct sums of countable groups (Kolettis, 1960 [10]), (3) totally projective groups (Hill, 1967 [5]), and (4) $S$-groups (Warfield, 1975 [18]). Other significant contributions to the classification problem include Zippin [20], Nunke [13], Hill and Megibben [8], Parker and Walker [15], and Crawley and Hales [1]; we do not claim to be exhaustive, but our intent is only to provide a reasonable bibliography. Moreover, our interest here is restricted to $p$-groups. For the classification of (certain classes of) mixed groups, see [14, 17, 19], or other sources.

Finally, we mention that the tendency upon reaching each of the milestones referenced above has been at first to perceive the situation as being as far as one can go. This is evidenced, for example, by the fact that a quarter of a century elapsed between Ulm's result for countable groups and Kolettis' extension to direct sums of such groups. Also, recall that Warfield initially thought that Problem 4 had a negative answer. My opinion is that no time soon will all $p$-groups be satisfactorily classified with numerical invariants, but it is inevitable that larger and larger classes will be reached with and encompassed in such a classification theory.

\section{The reduction to cofinality greater than $\omega_{0}$.}

THEOREM 1. Let $\mu$ denote an arbitrary limit ordinal. The class $A_{\mu}$ consists exclusively of totally projective groups if and only if $\mu$ is cofinal with $\omega_{0}$. 
Proof. If $\mu$ is not cofinal with $\omega_{0}$, it is well known that there exists totally projective $p$-groups $G$ of length $\mu$ with proper subgroups $H$ that are isotype and dense in the sense that $\left\langle p^{\lambda} G, H\right\rangle=G$ whenever $\lambda<\mu$. Moreover, it is known that any such subgroup $H$ cannot be totally projective. However, such an $H$ belongs to the class $A_{\mu}$. In other words, there is an $S$-group belonging to $A_{\mu}$ that is not totally projective [18].

It remains to show that if $\operatorname{cof}(\mu)=\omega_{0}$ then $H$ is totally projective for any $A_{\mu}$-pair $(H, G)$. In order to prove that such an $H$ is totally projective, let $\mathscr{C}$ be a collection of nice subgroups of $G$ that satisfy the third axiom of countability. Likewise let $\overline{\mathscr{C}}$ be a collection of nice subgroups of $(G / H) p^{\mu}(G / H)$ that satisfy the third axiom of countability. If $N$ is a subgroup of $G$, let $\bar{N}$ denote the image of $N$ under the natural $\operatorname{map} G \rightarrow(G / H) / p^{\mu}(G / H)$.

As in earlier papers, we say that two subgroups $A$ and $B$ of $G$ are compatible, in which case we write $A \| B$, if for every pair $(a, b) \in A \times B$ there exists $c$ in $A \cap B$ such that $h_{G}(a+c) \geqslant h_{G}(a+b)$. We want to consider those subgroups $N$ of $G$ that satisfy the following conditions:

(i) $N \in \mathscr{C}$.

(ii) $\bar{N} \in \overline{\mathscr{C}}$.

(iii) $N \| H$.

Claim. If $N$ satisfies (i)-(iii) and $C$ is any countable subgroup of $G$, there exists a countable subgroup $K$ of $G$ such that $\langle N, K\rangle \supseteq\langle N, C\rangle$ and such that the countable extension $N^{\prime}=\langle N, K\rangle$ of $N$ also satisfies conditions (i)-(iii).

For the moment, assume that the claim is valid. Since $\mathscr{C}$ and $\overline{\mathscr{C}}$ both satisfy the third axiom of countability and (iii) is an inductive property, we conclude from this that there is an ascending chain

$$
0=N_{0} \subseteq N_{1} \subseteq \cdots \subseteq N_{\alpha} \subseteq \cdots \subseteq G \quad(\alpha<\sigma)
$$

of subgroups $N_{\alpha}$ of $G$ satisfying conditions (i)-(iii) with the property that $G=$ $\bigcup_{\alpha<\sigma} N_{\alpha}, N_{\beta}=\bigcup_{\alpha<\beta} N_{\alpha}$ if $\beta$ is a limit less than $\sigma$, and $N_{\alpha+1} / N_{\alpha}$ is countable for each $\alpha$. It follows quickly that if we let $M_{\alpha}=N_{\alpha} \cap H$, then

$$
0=M_{0} \subseteq M_{1} \subseteq \cdots \subseteq M_{\alpha} \subseteq \cdots \quad(\alpha<\sigma)
$$

is a chain of nice subgroups of $H$. Thus $H$ satisfies the third axiom of countability [4], and the theorem is proved if we can validate the claim.

In order to verify the claim, it is enough to show only that there exists a countable group $B \supseteq C$ such that for each triple $(n, c, h) \in N \times C \times H$ there exists $x \in$ $\langle N, B\rangle \cap H$ for which $h_{G}(n+c+x) \geqslant h_{G}(n+c+h)$. For if we can establish the above, then upon setting $C_{0}=C$ and $C_{1}=B$ and inductively replacing $C_{i}$ by $C_{i+1}$, we can obtain an ascending sequence of countable subgroups $C_{0} \subseteq C_{1} \subseteq \cdots \subseteq C_{n}$ $\subseteq \cdots$ with the property that if $\left(n, c_{n}, h\right) \in N \times C_{n} \times H$ there exists $x \in\left\langle N, C_{n+1}\right\rangle$ $\cap H$ such that $h_{G}\left(n+c_{n}+x\right) \geqslant h_{G}\left(n+c_{n}+h\right)$. If we let $K=\cup C_{n}$, then for each $(n, k, h) \in N \times K \times H$ there exists $x \in\langle N, K\rangle \cap H$ such that $h_{G}(n+k+x) \geqslant$ $h_{G}(n+k+h)$, which shows that $\langle N, K\rangle \| H$. Since the subgroups $C_{n}, n \geqslant 1$, can be chosen so that $C_{n} \in \mathscr{C}$ and $\bar{C}_{n} \in \mathscr{C}$, we can construct $K=\bigcup C_{n}$ so that $K \in \mathscr{C}$ and 
$\bar{K} \in \overline{\mathscr{C}}$. Consequently, $\langle N, K\rangle \in \mathscr{C}$ and $\langle\bar{N}, \bar{K}\rangle \in \overline{\mathscr{C}}$. Therefore, $N^{\prime}=\langle N, K\rangle$ satisfies (i)-(iii). Thus the claim will be validated (and the theorem proved) if we can merely show that for each countable subgroup $C$ there is a countable subgroup $B \supseteq C$ such that, for each triple $(n, c, h) \in N \times C \times H$, there exists $x \in\langle N, B\rangle \cap$ $H$ such that $h_{G}(n+c+x) \geqslant h_{G}(n+c+h)$. We remark that it would be easy to produce such a subgroup $B$ if $N$ were also countable, so the uncountability of $N$ is what makes the problem nontrivial. Nevertheless, we shall produce such a countable subgroup $B$. List the elements of $C$ (using the positive integers for indices) as $c_{1}, c_{2}, \ldots, c_{i}, \ldots$. First, we deal with each $c_{i}$ individually. To each $c_{i}$ we associate at most a countable number of elements $h_{i, k}$ (or $h_{i}$ in case only a single element is required) in $H$ as follows:

Case 1. $\bar{c}_{i}=c_{i}+H+p^{\mu}(G / H) \in \bar{N}$. Choose $m_{i} \in N$ so that

$$
c_{i}+H+p^{\mu}(G / H)=m_{i}+H+p^{\mu}(G / H) .
$$

Since $\mu$ is cofinal with $\omega_{0}$, there exists an ascending sequence $\mu(k)$ of ordinals less than $\mu$ with $\sup \{\mu(k)\}=\mu$. Since $p^{\mu(k)}(G / H)=\left\langle p^{\mu(k)} G, H\right\rangle / H$ for each $k \geqslant 1$, there exists $h_{i, k} \in H$ such that

$$
m_{i}-c_{i}+h_{i, k} \in p^{\mu(k)} G .
$$

The countable collection of elements $h_{i, k} \in H$ are associated with $c_{i}$.

Case 2. $\bar{c}_{j} \notin \bar{N}$. Since $\bar{N}$ is nice in $(G / H) / p^{\mu}(G / H)$, there exist $m_{j} \in N$ so that the element $\bar{c}_{j}+\bar{m}_{j}$ is proper, that is, has maximal height in $(G / H) / p^{\mu}(G / H)$ among the elements of the coset $\bar{c}_{j}+\bar{N}$. Moreover, since $\bar{c}_{j} \notin \bar{N}$, the height of $\bar{c}_{j}+\bar{m}_{j}$ in $(G / H) / p^{\mu}(G / H)$ must be less than $\mu$, say $\lambda$. Since

$$
p^{\lambda}\left((G / H) / p^{\mu}(G / H)\right)=\left(p^{\lambda}(G / H)\right) / p^{\mu}(G / H)
$$

and

$$
p^{\lambda}(G / H)=\left\langle p^{\lambda} G, H\right\rangle / H,
$$

it follows for some $h_{j} \in H$ that $c_{j}+m_{j}+h_{j} \in p^{\lambda} G$. The single element $h_{j} \in H$ is associated with $c_{j}$.

Define $B=\left\langle C, h_{j}, h_{i, k}\right\rangle$, where $i$ and $j$ range over the appropriate subsets of the positive integers and $k \geqslant 1$. In order to show that $B$ has the desired property, suppose $(n, c, h) \in N \times C \times H$ and $h_{G}(n+c+h)=\alpha$. If $\alpha \geqslant \mu$, then $n+c+h$ $=0$ since $p^{\mu} G=0$. In this case we can take $x=h$, and we have $h_{G}(n+c+x) \geqslant$ $h_{G}(n+c+h)$ with $x \in\langle N, B\rangle \cap H$, since $h \in\langle N, C\rangle \subseteq\langle N, B\rangle$. Therefore, we may assume that $\alpha<\mu$. If $c=c_{i}$, where Case 1 holds for $c$, we know that $\mu(k)>\alpha$ if $k$ is chosen sufficiently large. Whence

$$
m_{i}-c_{i}+h_{i, k} \in p^{\alpha} G,
$$

and, consequently, $n+m_{i}+h+h_{i, k} \in p^{\alpha} G$. This implies that $n+m_{i}+x \in p^{\alpha} G$ for some $x \in N \cap H$ since $N \| H$. However, the latter implies, in turn, that

$$
n+c_{i}+\left(x-h_{i, k}\right) \in p^{\alpha} G .
$$

Since $c=c_{i}$ and $x-h_{i, k} \in\langle N, B\rangle \cap H$, the desired result holds. 
If $c=c_{j}$ where Case 2 holds for $c$, the argument is similar but is based on the relation

$$
c_{j}+m_{j}+h_{j} \in p^{\lambda} G
$$

instead of $m_{i}-c_{i}+h_{i, k} \in p^{\alpha} G$. In the present case, however, we need to observe that $\lambda \geqslant \alpha$. But $h_{G}(n+c+h)=\alpha$ certainly implies that $\bar{c}+\bar{N}$ has height $\alpha$, so $\lambda \geqslant \alpha$. This completes the proof of the theorem.

3. $A$-groups and $A$-invariants. Let $H$ be an $A$-group, and let $H=\sum \oplus H_{i}$ and $G=\sum \oplus G_{i}$, where $\left(H_{i}, G_{i}\right)$ is an $A_{\mu(i)}$-pair for distinct limit ordinals $\mu(i)$ that are not cofinal with $\omega_{0}$. We define what we call the $A$-numbers ( $=A$-invariants) of $H$ as follows. Actually we define a sequence of ordinal-to-cardinal functions $F_{\mu}$. Although the values of these functions should be the $A$-numbers, to simplify the terminology and to follow tradition, we often refer to the functions $F_{\mu}$ themselves as the $A$-numbers or $A$-invariants. As a preliminary step toward defining the functions $F_{\mu}$, set

$$
E_{\mu}=\bigcap_{\substack{\lambda<\mu \\ \lambda+\sigma=\mu}} p^{\sigma}\left(\left\langle p^{\lambda} G, H\right\rangle / H\right) /\left\langle p^{\mu} G, H\right\rangle / H
$$

whenever $\mu$ is a limit ordinal not cofinal with $\omega_{0}$. Now, define $F_{\mu}=F_{\mu}^{H}$, in case $\mu=0$, or $\mu$ is a limit ordinal not cofinal with $\omega_{0}$, by

$$
F_{\mu}(\alpha)= \begin{cases}\operatorname{dim}\left(p^{\alpha} H[p] / p^{\alpha+1} H[p]\right) & \text { if } \mu=0 \text { and } \alpha<\infty, \\ \operatorname{dim}\left(p^{\alpha} E_{\mu}[p] / p^{\alpha+1} E_{\mu}[p]\right) & \text { if } \mu \neq 0 \text { and } \alpha<\infty \\ \operatorname{dim}\left(p^{\alpha} E_{\mu}[p]\right) & \text { if } \mu \neq 0 \text { and } \alpha=\infty\end{cases}
$$

We remark that there is no loss of generality in assuming, as we have done, that $H$ and $G$ are reduced. Hence, $p^{\infty} H=0$, where $p^{\infty} A$ denotes the divisible part of a $p$-primary abelian group $A$, and there is no need to make a provision for $\alpha=\infty$ in case $\mu=0$. We also mention the obvious: $F_{0}(\alpha)$ is just the $\alpha$ th Ulm-Kaplansky invariant of $H$. Further, it is not difficult to show, for a limit $\mu$, that $p^{\sigma}$ can be deleted from the definition of $E_{\mu}$ without altering its meaning. However, aside from its symmetry, the above definition appears to make $E_{\mu}$ smaller (a desirable feature) while yet retaining a measure of how far $H$ is from being nice at the ordinal $\mu$. More importantly, we will discover (in Lemma B) a more intrinsic characterization of $E_{\mu}$, which can (and later will) be used as an alternate definition.

Lemma A. Let $H$ be an A-group, and let $H=\sum \oplus H_{i}$ and $G=\sum \oplus G_{i}$, where $\left(H_{i}, G_{i}\right)$ is an $A_{\mu(i)}$-pair for distinct limit ordinals $\mu(i)$ that are not cofinal with $\omega_{0}$. If $F_{\mu}(\alpha)$ is as defined above and $\mu \neq 0$, then $F_{\mu}(\alpha)=0$ unless $\mu=\mu(i)$ for some $i$. In case $\mu=\mu(i)$ for some $i$, then $F_{\mu}(\alpha)$ is the $\alpha$ th Ulm-Kaplansky invariant of $p^{\mu}\left(G_{i} / H_{i}\right)$ when $\alpha<\infty$, and $F_{\mu}(\infty)$ is the rank of the divisible part of this group.

Proof. Suppose $\mu \neq 0$. Let $\mu$ denote any limit ordinal not cofinal with $\omega_{0}$. According to the definition of $F_{\mu}(\alpha)$, if $\alpha<\infty$,

$$
F_{\mu}(\alpha)=\operatorname{dim}\left(p^{\alpha} E_{\mu}[p] / p^{\alpha+1} E_{\mu}[p]\right) .
$$


Since $E_{\mu}=\bigcap_{\lambda<\mu, \lambda+\sigma=\mu} p^{\sigma}\left(\left\langle p^{\lambda} G, H\right\rangle / H\right) /\left\langle p^{\mu} G, H\right\rangle / H$ and since $G=\sum \oplus G_{i}$ with the length of $G_{i}$ not exceeding $\mu(i)$, it is clear that those summands $G_{i}$ with $\mu(i)<\mu$ make no contributions to $E_{\mu}$ (and therefore make no contribution to $F_{\mu}(\alpha)$ ). Moreover, if $\mu(i)>\mu$ and $\lambda+\sigma=\mu$, then

$$
p^{\sigma}\left(\left\langle p^{\lambda} G_{i}, H_{i}\right\rangle / H_{i}\right)=p^{\sigma} p^{\lambda}\left(G_{i} / H_{i}\right)=p^{\mu}\left(G_{i} / H_{i}\right)=\left\langle p^{\mu} G_{i}, H_{i}\right\rangle / H_{i} .
$$

Thus, $G_{i}$ again makes no contribution to $E_{\mu}$, so $E_{\mu}=0$ unless $\mu=\mu(i)$ for some $i$. If $\mu=\mu(i)$, then

$$
E_{\mu}=\bigcap_{\substack{\lambda<\mu \\ \lambda+\sigma=\mu}} p^{\sigma}\left(\left\langle p^{\lambda} G_{i}, H_{i}\right\rangle / H_{i}\right) /\left\langle p^{\mu} G_{i}, H_{i}\right\rangle / H_{i}=p^{\mu}\left(G_{i} / H_{i}\right) .
$$

This completes the proof of Lemma A.

Lemma B. Assume the same notation and hypothesis as in Lemma A, and let $\mu$ be a limit ordinal not cofinal with $\omega_{0}$. Let $H_{\mu}=H / p^{\mu} H$ and let $\bar{H}_{\mu}$ denote the completion of $H_{\mu}$ in the $p^{\mu}$-topology (having $p^{\lambda} H / p^{\mu} H, \lambda<\mu$, as a base for the neighborhoods of zero). Then $E_{\mu}=\bar{H}_{\mu} / H_{\mu}$. In particular, the A-numbers of $H$ are invariants of $H$.

Proof. Since $G$ is totally projective and $\mu$ is a limit ordinal not cofinal with $\omega_{0}$, $G / p^{\mu} G=\sum \oplus G_{i} / p^{\mu} G_{i}$ is Hausdorff and complete in its $p^{\mu}$-topology. Since $H / p^{\mu} H$ $=\sum \oplus H_{i} / p^{\mu} H_{i}$ is isotype in $G / p^{\mu} G$, the completion of $H / p^{\mu} H$ is simply its closure in $G / p^{\mu} G$. But from what has gone before, it follows that $H / p^{\mu} H$ is closed in $G / p^{\mu} G$ unless $\mu=\mu(i)$ for some $i$. Moreover, in case $\mu=\mu(i)$, we have $\bar{H}_{\mu} / H_{\mu}=$ $p^{\mu}\left(G_{i} / H_{i}\right)=E_{\mu}$, with $\bar{H}_{\mu}$ being the closure of $H_{\mu}=H / p^{\mu} H$ in $G / p^{\mu} G$.

Theorem 2. Let $H$ and $H^{\prime}$ be A-groups, and let $H=\sum \oplus H_{i}, H^{\prime}=\sum \oplus H_{i}^{\prime}$, $G=\sum \oplus G_{i}$, and $G^{\prime}=\sum \oplus G_{i}^{\prime}$, where $\left(H_{i}, G_{i}\right)$ and $\left(H_{i}^{\prime}, G_{i}^{\prime}\right)$ are $H_{\mu(i)}$-pairs for distinct limit ordinals $\mu(i)$ not cofinal with $\omega_{0}$. If $H$ and $H^{\prime}$ have the same A-numbers, there exists an isomorphism from $p^{\mu(i)}\left(G_{i} / H_{i}\right)$ onto $p^{\mu(i)}\left(G_{i}^{\prime} / H_{i}^{\prime}\right)$ for each $i$.

Proof. We know that $E_{\mu(i)}$ and $E_{\mu(i)}^{\prime}$ have the same Ulm-Kaplansky invariants and their divisible parts are isomorphic. By Lemma A, $E_{\mu(i)}=p^{\mu(i)}\left(G_{i} / H_{i}\right)$ and $E_{\mu(i)}^{\prime}=p^{\mu(i)}\left(G_{i}^{\prime} / H_{i}^{\prime}\right)$. Thus, the reduced parts of $E_{\mu}$ and $E_{\mu}^{\prime}$ are totally projective. Therefore, since they have the same Ulm-Kaplansky invariants, $E_{\mu}$ and $E_{\mu(i)}^{\prime}$ must be isomorphic, and the theorem is proved.

Lemma C. Suppose that $\left(H_{i}, G_{i}\right)$ and $\left(H_{i}^{\prime}, G_{i}^{\prime}\right)$ are $A_{\mu(i)}$-pairs for distinct ordinals $\mu(i)$. Further, suppose that $\phi_{i}: G_{i} / H_{i} \nrightarrow G_{i}^{\prime} / H_{i}^{\prime}$ is an isomorphism between corresponding quotients. Let $G=\sum \oplus G_{i}, G^{\prime}=\sum \oplus G_{i}^{\prime}, H=\sum \oplus H_{i}$, and $H^{\prime}=\sum \oplus H_{i}^{\prime}$. Finally, let $\phi=\sum \phi_{i}$ denote the natural isomorphism from $G / H$ onto $G^{\prime} / H^{\prime}$ determined by the $\phi_{i}$. If $g \in G$ but $g \notin H$ and $\phi(g+H)=g^{\prime}+H^{\prime}$, there exists $h^{\prime} \in H^{\prime}$ such that $h_{G^{\prime}}\left(g^{\prime}+h^{\prime}\right) \geqslant h_{G}(g)$. Moreover, if $h_{G / H}(g+H)>h_{G}(g)$, there exists $h^{\prime} \in H^{\prime}$ such that $h_{G^{\prime}}\left(g^{\prime}+h^{\prime}\right)>h_{G}(g)$.

Proof. Since $h_{G}(g)=\min \left\{h_{G_{i}}\left(g_{i}\right)\right\}$ if $g=\sum g_{i}$, with $g_{i} \in G_{i}$, it suffices to prove the lemma for the $i$ th component. However, for this special case the lemma is almost an immediate consequence of $\left(H_{i}, G_{i}\right)$ and $\left(H_{i}^{\prime}, G_{i}^{\prime}\right)$ being $A_{\mu(i)}$-pairs. Specifically, if 
$h_{G_{i}}\left(g_{i}\right)=\alpha$ then $\alpha<\mu(i)$ provided that $g_{i} \in G_{i} \backslash H_{i}$. Therefore,

$$
g_{i}^{\prime}+H^{\prime} \in p^{\alpha}\left(G_{i}^{\prime} / H_{i}^{\prime}\right)=\left\langle p^{\alpha} G_{i}^{\prime}, H_{i}^{\prime}\right\rangle / H_{i}^{\prime}
$$

and

$$
g_{i}^{\prime}+H_{i}^{\prime} \in p^{\alpha+1}\left(G_{i}^{\prime} / H_{i}^{\prime}\right)=\left\langle p^{\alpha+1} G_{i}^{\prime}, H_{i}^{\prime}\right\rangle / H_{i}^{\prime}
$$

if $g_{i}+H_{i} \in p^{\alpha+1}\left(G_{i} / H_{i}\right)$. This completes the proof of the Lemma.

\section{Uniqueness theorem.}

THEOREM 3. If $H$ and $H^{\prime}$ are $A$-groups with the same $A$-numbers, then $H$ and $H^{\prime}$ are isomorphic.

Proof. For distinct ordinals $\lambda(i)$ not cofinal with $\omega_{0}$ let $\left(H_{i}, G_{i}\right)$ and $\left(H_{i}^{\prime}, G_{i}^{\prime}\right)$ be $A_{\lambda(i)}$-pairs, where $H=\Sigma \oplus H_{i}$ and $H^{\prime}=\sum \oplus H_{i}$. There is no loss of generality in assuming the index set is the same for both since, for example, we can add, if necessary, $A_{\lambda(i)}$-pairs of the type $\left(0, G_{i}\right)$ or $\left(0, G_{i}^{\prime}\right)$ without changing $H$ or $H^{\prime}$. Since $H$ and $H^{\prime}$ have the same $A$-numbers, $p^{\lambda(i)}\left(G_{i} / H_{i}\right) \cong p^{\lambda(i)}\left(G_{i}^{\prime} / H_{i}^{\prime}\right)$ according to Theorem 2. There is no loss of generality in assuming that $G_{i}=G_{i}^{\prime}$ and $G_{i} / H_{i} \cong$ $G_{i}^{\prime} / H_{i}^{\prime}$, for we can replace both $G_{i}$ and $G_{i}^{\prime}$ by

$G_{i} \oplus G_{i}^{\prime} \oplus \sum_{\aleph_{0}} \oplus\left\{G_{i} \oplus G_{i}^{\prime} \oplus\left\{\left(G_{i} / H_{i}\right) / p^{\lambda(i)}\left(G_{i} / H_{i}\right) \oplus\left(G_{i}^{\prime} / H_{i}^{\prime}\right) / p^{\lambda(i)}\left(G_{i}^{\prime} / H_{i}^{\prime}\right)\right\}\right\}$.

Thus, to simplify notation we shall make this assumption. Indeed, we may assume (without loss of generality) that $G_{i}=G_{i}^{\prime}$ and $G_{i} / H_{i} \cong G_{i}^{\prime} / H_{i}^{\prime}$. Let $\phi_{i}: G_{i} / H_{i} \nrightarrow$ $G_{i} / H_{i}^{\prime}$ be an isomorphism, and set $\phi=\sum \phi_{1}$. Since $G_{i}=G_{i}^{\prime}, G=\sum \oplus G_{i}=\sum \oplus G_{i}^{\prime}$ $=G^{\prime}$. Moreover, $\phi$ is an isomorphism from $G / H$ onto $G / H^{\prime}$ that maps the $i$ th component $G_{i} / H_{i}$ onto the corresponding $i$ th component $G_{i} / H_{i}{ }^{\prime}$. Thus, Lemma $\mathrm{C}$ is applicable here since its hypotheses are satisfied.

We claim that there is an automorphism of $G$ that maps $H$ onto $H^{\prime}$. In the original draft of this paper we included a detailed proof of this fact. However, since there are now two other proofs that are available we have suppressed these details. Our original proof was virtually the same as the proof of the uniqueness theorem for $N$-groups in [6] with the essential difference between the two proofs being reconciled by Lemma C. Hence, we simply offer this reference as one proof. A second (and much shorter) proof can be obtained as follows. Subsequent to the original proof, C. Megibben and I have proved the following

THeOREM (HILl AND MEgIBBEN [9]). If $H$ and $H^{\prime}$ are isotype subgroups of a totally projective group $G$, then $H$ and $H^{\prime}$ are isomorphic provided they have the same Ulm-Kaplansky invariants and $G / H$ and $G / H^{\prime}$ are isomorphic as valuated groups endowed with the coset valuation.

The coset valuation of $G / H$ is defined by

$$
|g+H|=\sup \{|g+h|+1: h \in H\} \text {. }
$$

It can be quickly verified that the isomorphism $\phi_{i}: G_{i} / H_{i} \nrightarrow G_{i} H_{i}^{\prime}$ defined above preserves the coset valuation. Therefore, so does $\phi=\sum \phi_{i}$, and the theorem that 
$H \cong H^{\prime}$ is a consequence, at this point of the argument, of the theorem cited by Hill and Megibben.

5. Existence theorem. Although not called by that name, isolated examples of $A$-groups (even before the advent of $N$-groups) can be found in the literature that are not totally projective nor $S$-groups. One of the most notable cases is an example studied by Megibben in [11]. We suspect, in fact, that Megibben came close at that time to discovering the class of $N$-groups, if not $A$-groups, and their significance. As we have mentioned before, $N$-groups are a special case of $A$-groups. They were classified in [6], where a general existence theorem, as well as a uniqueness theorem, was established for them. Our purpose in this section is to establish the corresponding existence theorem for $A$-groups. Toward that effort we first take care of some preliminaries. As we shall see, the situation is much more complicated for $A$-groups.

Let $f$ be an ordinal-to-cardinal function that eventually vanishes (for sufficiently large ordinals). If one prefers, the function can be restricted to an initial segment of the ordinals. We frequently adjoin $\infty$ to the ordinals and permit it to be in the domain of $f$ with $f(\infty)$ not required to be zero (although $\infty>\alpha$ if $\alpha$ is an ordinal). If $\alpha<\beta \leqslant \infty$, define

$$
\int_{\alpha}^{\beta} f(\gamma)=\sum_{\alpha \leqslant \gamma<\beta} f(\gamma)
$$

Note that we conventionally integrate (or sum if one prefers) over a half-open interval. However, when it is desirable to integrate over a closed interval, we set

$$
\int_{\alpha}^{\beta+} f(\gamma)=\sum_{\alpha \leqslant \gamma \leqslant \beta} f(\gamma)=\left(\int_{\alpha}^{\beta} f(\gamma)\right)+f(\beta) ;
$$

in particular, $\int_{\alpha}^{\infty+} f(\gamma)=\left(\int_{\alpha}^{\infty} f(\gamma)\right)+f(\infty)$.

The function $f$ is said to be admissible if

$$
\int_{\alpha}^{\alpha+\omega} f(\gamma)=\int_{\alpha}^{\infty} f(\gamma)
$$

for every ordinal $\alpha$; compare with [2, p. 70]. A standard example of an admissible function $f$ is the Ulm-Kaplansky function associated with a totally projective group $G$, defined (for any $G$ ) by

$$
f(\gamma)=\operatorname{dim}\left(p^{\gamma} G[p] / p^{\gamma+1} G[p]\right) .
$$

If there is a need for a distinction, we call the one-point extension of $f$ given by $f(\infty)=p^{\infty} G[p]$ the extended Ulm-Kaplansky function of $G$, which is relevant only when $G$ is not reduced.

Throughout this section, it is understood that all functions considered, unless otherwise specified, are ordinal-to-cardinal functions. The length of such a function $f$ is the smallest ordinal $\lambda$ for which $f(\gamma)=0$ whenever $\gamma \geqslant \lambda$ and $\gamma \neq \infty$. For example, the length of the extended Ulm-Kaplansky function of any p-group $G$ is 
the length of the reduced part of $G$. Another concept that will be used is the following

Definition. If $f$ and $g$ are functions and $f$ is of length $\mu>0$, we say that $f$ totally dominates $g$ if

$$
\int_{\lambda}^{\infty} f(\gamma) \geqslant \int_{0}^{\infty+} g(\gamma)
$$

whenever $\lambda<\mu$.

Before we are prepared to prove the general existence theorem for $A$-groups, certain combinatorial results are needed. These are provided in the next two lemmas. Since $\omega_{0}$ appears frequently in this section, we let $\omega=\omega_{0}$. Likewise, $\operatorname{cof}(\mu)$ denotes the cofinality of a limit ordinal $\mu$, and a cardinal is identified with the smallest ordinal of the given cardinality.

LEMMA D. Let $f$ and $g_{\mu}, \mu \in \mathscr{M}$, be nonzero admissible functions; where $\mathscr{M}$ is a collection of limit ordinals not cofinal with $\omega$. If

$$
\int_{\lambda}^{\infty} f(\alpha) \geqslant \sum_{\mu>\lambda} \int_{0}^{\infty+} g_{\mu}(\alpha)
$$

for each ordinal $\lambda$ (that satisfies $\mu>\lambda$ for some $\mu \in \mathscr{M}$ ), there exist functions $f_{\mu}$, $\mu \in \mathscr{M}$, that satisfy the following conditions:

(a) $f_{\mu}$ is admissible of length $\mu$.

(b) $f_{\mu}$ totally dominates $g_{\mu}$ (that is, $\int_{\lambda}^{\mu} f_{\mu}(\alpha) \geqslant \int_{0}^{\infty+} g_{\mu}(\alpha)$ when $\lambda<\mu$ ).

(c) $f(\alpha)=f(\alpha)+\sum_{\mu \in \mathscr{M}} f_{\mu}(\alpha)$ for each $\alpha$.

Proof. For each limit ordinal $\lambda$, let $\mathscr{M}_{\lambda}=\{\mu \in \mathscr{M}: \mu>\lambda\}$. We define $f_{\mu}$, for all $\mu \in \mathscr{M}$, on the half-open interval $[\lambda, \lambda+\omega)$ as follows. If $\mu \leqslant \lambda$ or, equivalently, if $\mu \notin \mathscr{M}_{\lambda}$, then $f_{\mu}(\alpha)=0$ for every $\alpha$ in $[\lambda, \lambda+\omega)$; in particular, if $\mathscr{M}_{\lambda}$ is vacuous then $f_{\mu}(\alpha)=0$ when $\alpha \geqslant \lambda$ for each $\mu \in \mathscr{M}$. We now deal with those $\mu$ in $\mathscr{M}_{\lambda}$. Assume that $\mathscr{M}_{\lambda}$ is nonempty. Two cases are distinguished: in both cases it is understood always that $\mu \in \mathscr{M}_{\lambda}$ and $\alpha \in[\lambda, \lambda+\omega)$.

Case 1. $f(\alpha) \geqslant\left|\mathscr{M}_{\lambda}\right|$ for infinitely many $\alpha$ (between $\lambda$ and $\left.\lambda+\omega\right)$. Define

$$
f_{\mu}(\alpha)= \begin{cases}f(\alpha) & \text { if } f(\alpha) \geqslant\left|\mathscr{M}_{\lambda}\right| \boldsymbol{\kappa}_{0}, \\ 0 & \text { otherwise. }\end{cases}
$$

Since $\int_{\lambda+n}^{\lambda+\omega} f(\alpha)=\int_{\lambda+n}^{\infty} f(\alpha) \geqslant \operatorname{cof}(\mu)$ when $\mu \in \mathscr{M}_{\lambda}$ and since $\operatorname{cof}(\mu)>\boldsymbol{\aleph}_{0}$, we immediately see that Case 1 yields $f_{\mu}(\alpha)=f(\alpha)$ for infinitely many $\alpha$; in other words, the condition $f(\alpha) \geqslant\left|\mathscr{M}_{\lambda}\right|$ for infinitely many $\alpha$ is equivalent to the condition $f(\alpha) \geqslant\left|\mathscr{M}_{\lambda}\right| \boldsymbol{N}_{0}$ for infinitely many $\alpha$. Moreover, it is easy to deduce that

$$
\int_{\lambda+n}^{\lambda+\omega} f_{\mu}(\alpha)=\int_{\lambda+n}^{\lambda+\omega} f(\alpha)
$$

whenever $n<\omega$.

Case 2. $f(\alpha)<\left|\mathscr{M}_{\lambda}\right|$ for all but a finite number of $\alpha$. Note that this precludes the countability of $\mathscr{M}_{\lambda}$. Since $\int_{\lambda+n}^{\lambda+\omega} f(\alpha) \geqslant\left|\mathscr{M}_{\lambda}\right|$, for each $n<\omega$, it is clear that $\left|\mathscr{M}_{\lambda}\right|$ must be cofinal with $\omega$. Thus $\mathscr{M}_{\lambda}$ is the union of a countable number of disjoint infinite subsets $S_{n}$ smaller than $\mathscr{M}_{\lambda}$; assume, without loss of generality, that 
$\left|S_{i}\right|<\left|S_{2}\right|<\cdots<\left|S_{n}\right|<\cdots$. This partition of $\mathscr{M}_{\lambda}$ is arbitrary (but fixed), and its sole purpose is to contribute toward the definition of $f_{\mu}(\alpha)$ on the interval $[\lambda, \lambda+\omega)$. Indeed, we define $f_{\mu}(\alpha)$, for $\mu \in S_{n}$, by

$$
f_{\mu}(\alpha)= \begin{cases}f(\alpha) & \text { if } f(\alpha) \geqslant\left|S_{n}\right| \\ 0 & \text { otherwise }\end{cases}
$$

Observe that, as in Case $1, f_{\mu}(\alpha)=f(\alpha)$ for infinitely many $\alpha$. Moreover, $\int_{\lambda+k}^{\lambda+\omega} f_{\mu}(\alpha)$ $=\int_{\lambda+k}^{\lambda+\omega} f(\alpha)$ whenever $k<\omega$ (because $f(\alpha)$ and $f_{\mu}(\alpha)$ disagree only if $f_{\mu}(\alpha)=0$, in which case $f(\alpha)$ is relatively small).

The two cases no longer need distinguishing. It is a quick deduction that

$$
f(\alpha)=f(\alpha)+\sum_{\mu \in \mathscr{M}_{\lambda}} f_{\mu}(\alpha)
$$

for each $\alpha$ in the interval $[\lambda, \lambda+\omega)$. Since $f_{\mu}(\alpha)=0$ if $\mu \notin \mathscr{M}_{\lambda}$, certainly condition (c) holds, first on the interval $[\lambda, \lambda+\omega)$ and then on the whole domain of $f$. For $\lambda<\mu$, the inequalities

$$
\begin{gathered}
\int_{\lambda+n}^{\infty} f_{\mu}(\alpha) \geqslant \int_{\lambda+n}^{\lambda+\omega} f_{\mu}(\alpha)=\int_{\lambda+n}^{\lambda+\omega} f(x)=\int_{\lambda+n}^{\infty} f(\alpha) \\
\geqslant \sum_{\nu>\lambda} \int_{0}^{\infty+} g_{\nu}(\alpha) \geqslant \int_{0}^{\infty+} g_{\mu}(\alpha)
\end{gathered}
$$

demonstrate that $f_{\mu}$ totally dominates $g_{\mu}$. Thus, condition (b) is satisfied. Similarly, the comparison

$$
\int_{\lambda+n}^{\lambda+\omega} f_{\mu}(\alpha)=\int_{\lambda+n}^{\infty} f(\alpha) \geqslant \int_{\lambda+n}^{\infty} f_{\mu}(\alpha)
$$

verifies that $f_{\mu}$ is admissible. It is clear from its definition that $f_{\mu}$ has length not exceeding $\mu$, but the above inequalities for $\lambda<\mu$ imply that the length of $f_{\mu}$ is at least $\mu$. Therefore, condition (a) is satisfied and the lemma is proved.

Another result similar to the preceding lemma that will be useful in the proof of the existence theorem is the following

LEMMA E. For an arbitrary limit ordinal $\mu$, let $f$ be an admissible function of length $\mu$. Let $m$ be a fixed cardinal not cofinal with $\omega$ and suppose that $\int_{\lambda}^{\mu} f(\alpha) \geqslant m$ whenever $\lambda<\mu$. If $\mu(\tau)(\tau<m)$ is a collection of not more than $m$ limit ordinals less than $\mu$, then there exist functions $f_{\tau}(\tau<m)$ that satisfy the following conditions.

(1) $f_{\tau}$ is admissible of length $\mu(\tau)$.

(2) $\int_{\lambda}^{\lambda+\omega} f_{\tau}(\alpha)=\int_{\lambda}^{\lambda+\omega} f(\alpha)$ whenever $\lambda<\mu(\tau)$.

(3) $f_{\tau}(\alpha)=m f_{\tau}(\alpha)=\sum_{\rho \leqslant \tau} f_{\rho}(\alpha)$ for $\alpha<\mu(\tau)$.

(4) $f(\alpha)=f(\alpha)+\sum_{\tau<m} f_{\tau}(\alpha)$ for each $\alpha$.

Proof. Define the function $f_{\tau}$ as follows:

$$
f_{\tau}(\alpha)= \begin{cases}f(\alpha) & \text { if } \alpha<\mu(\tau) \text { and } f(\alpha) \geqslant m, \\ 0 & \text { otherwise. }\end{cases}
$$

Observe that if $\lambda<\mu$ then $f(\alpha) \geqslant m$ for infinitely many $\alpha$ between $\lambda$ and $\lambda+\omega$ because

$$
\int_{\lambda+n}^{\lambda+\omega} f(\alpha)=\int_{\lambda+n}^{\infty} f(\alpha) \geqslant m
$$


and $m$ is not cofinal with $\omega$. This leads at once to $\int_{\lambda}^{\lambda+\omega} f_{\tau}(\alpha)=\int_{\lambda}^{\lambda+\omega} f(\alpha)$ whenever $\lambda<\mu(\tau)$. The verification of conditions (1), (3), and (4) is now straightforward, and (2) is already settled.

THEOREM 4. Let $f$ and $g_{\mu}, \mu \in \mathscr{M}$, be nonzero admissible functions, where $\mathscr{M}$ is a collection of limit ordinals not cofinal with $\omega$. A necessary and sufficient condition for the existence of an A-group with A-invariants

$$
\begin{aligned}
& F_{0}(\alpha)=f(\alpha), \\
& F_{\mu}(\alpha)=g_{\mu}(\alpha) \quad \text { if } \mu \in \mathscr{M}, \\
& F_{\nu}(\alpha)=0 \quad \text { if } \nu \neq 0 \text { and } \nu \notin \mathscr{M},
\end{aligned}
$$

is, for each $\lambda$ (less than some $\mu \in \mathscr{M}$ ), the inequality

$$
(++) \quad \int_{\lambda}^{\lambda+\omega} f(\alpha) \geqslant \sum_{\mu>\lambda} \int_{0}^{\infty+} g_{\mu}(\alpha) .
$$

Proof. Sufficiency. Suppose $(++)$ holds. If $\mathscr{M}$ is empty, all that is required is a totally projective group $H$ whose Ulm-Kaplansky function is $f$. We know such an $H$ exists [5 or 2]. If $\mathscr{M}$ is nonempty, Lemma $\mathrm{D}$ reduces the proof to the case where $\mathscr{M}$ contains a single element $\mu$ and $f$ has length $\mu$. Indeed we can write, according to Lemma $\mathrm{D}, f=f+\sum_{\mu \in \mathscr{M}} f_{\mu}$, where $f_{\mu}$ is admissible of length $\mu$ and $f_{\mu}$ totally dominates $g_{\mu}$. As we have mentioned, there is certainly a totally projective group whose Ulm-Kaplansky function if $f$. Thus, it suffices to prove the existence of an $A$-group $H_{\mu}$ with $A$-invariants

$$
F_{0}(\alpha)=f_{\mu}(\alpha), \quad F_{\mu}(\alpha)=g_{\mu}(\alpha), \quad F_{\nu}(\alpha)=0 \quad \text { if } \nu \neq 0 \text { and } \nu \neq \mu .
$$

To simplify notation let $f=f_{\mu}$ and $g=g_{\mu}$. Recall that $f$ totally dominates $g$, that is,

$$
(+) \quad \int_{\lambda}^{\mu} f(\alpha) \geqslant \int_{0}^{\infty+} g(\alpha)
$$

for each $\lambda<\mu$.

Let the cofinality of $\mu$ be $\sigma>\omega$, and let $\mu(\tau), \tau<\sigma$, be a strictly increasing sequence of ordinals with limit $\mu$. Without loss of generality we may assume that $\mu(\tau)$ is a limit ordinal of the form $\gamma+\omega$ for every $\tau<\sigma$. In fact, we may assume that $\mu(\tau)=\lambda(\tau)+\omega$, where $\lambda(\tau)$ itself is of the form $\gamma+\omega$. Thus, choose $\mu(\tau)$ and $\lambda(\tau)$ in this manner. For convenience and agreement in notation with a previous lemma, set $m=|\sigma|=|\operatorname{cof}(\mu)|$ and note that $m \geqslant \boldsymbol{\aleph}_{1}$. Denote by $E$ the direct sum of a totally projective group and a divisible group that has $g$ for its extended Ulm-Kaplansky function. If we consider the function $f$, then clearly, $\int_{\lambda}^{\mu} f(\alpha) \geqslant m$ for any $\lambda<\mu$ since $f$ is admissible of length $\mu$. Observe, in fact, that condition (+) implies

$$
\int_{\lambda}^{\lambda+\omega} f(\alpha)=\int_{\lambda}^{\mu} f(\alpha) \geqslant m|E|
$$

in view of the inequality $|E| \leqslant \aleph_{0} \int_{0}^{\infty+} g(\alpha)$ for any (nonreduced) totally projective group $E$ with (extended) Ulm-Kaplansky function $g$. We now employ Lemma $E$ and write

$$
f(\alpha)=f(\alpha)+\sum_{\tau<\sigma} f_{\tau}(\alpha)
$$


where $f_{\tau}$ is an admissible function of length $\mu(\tau), \int_{\lambda}^{\lambda+\omega} f_{\tau}(\alpha)=\int_{\lambda}^{\lambda+\omega} f(\alpha)$ whenever $\lambda<\mu(\tau)$, and $f_{\tau}(\alpha)=m f_{\tau}(\alpha)=\sum_{\rho \leqslant \tau} f_{\rho}(\alpha)$. By our previous conclusion, observe that

$$
\int_{\lambda}^{\lambda+\omega} f_{\tau}(\alpha)=\int_{\lambda}^{\lambda+\omega} f(\alpha) \geqslant m|E|
$$

whenever $\lambda<\mu(\tau)$.

Let $B_{0} \leadsto B \rightarrow E$ be a pure-exact sequence where $B$ is a direct sum of cyclic groups and $|B|=|E|$. Denote by $C_{\tau}$ the totally projective group of length $\mu(\tau)=$ $\lambda(\tau)+\omega$ that has $f_{\tau}$ for its Ulm-Kaplansky function, and let $C_{\tau}^{\prime}$ be the totally projective group (of length at most $\mu(\tau)$ and at least $\lambda(\tau)$ ) that satisfies $p^{\lambda(\tau)} C_{\tau}^{\prime}=B$ and $C_{\tau}^{\prime} / p^{\lambda(\tau)} C_{\tau}^{\prime}=C_{\tau} / p^{\lambda(\tau)} C_{\tau}$. With $C_{\tau}^{\prime}$ so defined for each $\tau<\sigma$, we inductively define an ascending chain of totally projective groups $G_{\tau}$, for $\tau<\sigma$, as follows. Let $G_{0}=B$. If $G_{\alpha}$ has been defined for $\alpha \leqslant \tau<\sigma$ so that $G_{\alpha}$ is a totally projective group with $p^{\lambda} G_{\alpha}=B$ for some $\lambda \leqslant \lambda(\alpha)$ (and more particularly $p^{\lambda(\alpha)} G_{\alpha+1}=B$ whenever $\alpha<\tau$ ), define $G_{\tau+1}$ to be the pushout associated with $B \nrightarrow G_{\tau}$ and $B \nrightarrow C_{\tau}^{\prime}$. Thus we have the commutative diagram (with natural maps)

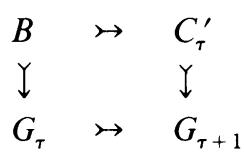

Alternately, we could say that $G_{\tau+1}$ is the amalgamated sum of $G_{\tau}$ and $C_{\tau}^{\prime}$. Observe that $p^{\lambda(\tau)} G_{\tau+1}=B=p^{\lambda(\tau)} C_{\tau}^{\prime}$ since

$$
G_{\tau+1} / B=G_{\tau} / B \oplus C_{\tau}^{\prime} / B
$$

and since $p^{\lambda(\tau)} G_{\tau} \subseteq B$ by the induction hypothesis. As expected, if $\tau$ is a limit less than $\sigma$ and if $G_{\alpha}$ has been defined for $\alpha<\tau$, we let $G_{\tau}=\cup_{\alpha<\tau} G_{\alpha}$ (where $G_{\alpha} \subseteq G_{\beta}$, if $\alpha<\beta$, by virtue of the natural identification of $G_{\alpha}$ in $\left.G_{\alpha+1}\right)$. By induction, it is apparent that

$$
G_{\tau} / B=\sum_{\gamma<\tau} \oplus C_{\gamma}^{\prime} / B
$$

and that $p^{\lambda} G_{\tau}=B$, where $\lambda=\sup _{\gamma<\tau}\{\lambda(\gamma)\} \leqslant \lambda(\tau)$. Thus, we have constructed a smooth $\left(G_{\beta}=\cup_{\alpha<\beta} G_{\alpha}\right.$ when $\beta$ is a limit), ascending chain of totally projective groups $G_{\tau}, \tau<\sigma$, with $p^{\lambda} G_{\tau}=B$ where $\lambda=\sup _{\gamma<\tau}\{\lambda(\gamma)\}$, and $G_{\tau} / B=\sum_{\gamma<\tau} \oplus$ $C_{\gamma}^{\prime} / B$. We can extend this chain one additional link and retain the same properties simply by defining $G_{\sigma}=\bigcup_{\tau<\sigma} G_{\tau}$. Note, in particular, that $p^{\mu} G_{\sigma}=B$ since $\mu=$ $\sup _{\tau<\sigma}\{\lambda(\tau)\}$. Indeed, if we set $A=G_{\sigma} / B_{0}$ then $p^{\mu} A=B / B_{0}=E$ and $A / p^{\mu} A=G_{\sigma} / B=\sum_{\tau<\sigma} \oplus C_{\tau}^{\prime} / B$. Therefore, $A / p^{\mu} A$ is the totally projective group whose Ulm-Kaplansky function is $\sum_{\tau<\sigma} f_{\tau}^{\prime}$, where

$$
f_{\tau}^{\prime}(\alpha)= \begin{cases}f_{\tau}(\alpha) & \text { if } \alpha<\lambda(\tau), \\ 0 & \text { otherwise. }\end{cases}
$$

Obviously, $f_{\tau}^{\prime}$ is the Ulm-Kaplansky function of $C_{\tau}^{\prime} / B=C_{\tau}^{\prime} / p^{\lambda(\tau)} C_{\tau}^{\prime}$. Observe that

$$
G_{\tau+1} / B=\sum_{\gamma \leqslant \tau} \oplus C_{\gamma}^{\prime} / B \cong C_{\tau}^{\prime} / B=C_{\tau} / p^{\lambda(\tau)} C_{\tau}
$$


because all are totally projective and have the same Ulm-Kaplansky invariants; recall that $m f_{\tau}(\alpha)=f_{\tau}(\alpha)=\sum_{\rho \leqslant \tau} f_{\rho}(\alpha)$.

Since $\int_{\lambda(\tau)+n}^{\mu(\tau)} f_{\tau}(\alpha) \geqslant m|E|$ and $\rho^{\lambda(\tau)} C_{\tau}$ is a direct sum of cyclic groups, there exists an epimorphism

$$
p^{\lambda(\tau)} C_{\tau} \rightarrow B=p^{\lambda(\tau)} G_{\tau+1} .
$$

Let $K_{\tau}$ denote its kernel. Due to the total projectivity of $C_{\tau} / K_{\tau} \cong G_{\tau+1}$, the isomorphism

$$
p^{\lambda(\tau)}\left(C_{\tau} / K_{\tau}\right)=p^{\lambda(\tau)} C_{\tau} / K_{\tau} \nrightarrow B=p^{\lambda(\tau)} G_{\tau+1}
$$

can be extended to an isomorphism between $C_{\tau} / K_{\tau}$ and $G_{\tau+1}$ (by virtue of Zippin's theorem for totally projectives). Denote by $\varepsilon_{\tau}$ the resulting epimorphism from $C_{\tau}$ to $G_{\tau+1}$,

$$
\varepsilon_{\tau}: C_{\tau} \rightarrow C_{\tau} / K_{\tau} \nrightarrow G_{\tau+1},
$$

and let

$$
\phi_{\tau}: C_{\tau} \rightarrow G_{\tau+1} \rightarrow G_{\tau+1} / B_{0} \subseteq A
$$

be the composition of $\varepsilon_{\tau}$ and the projection of $G_{\tau+1}$ onto $G_{\tau+1} / B_{0}$, which is a subgroup of $A$. Observe that if $\lambda<\mu$ and $a \in p^{\lambda} A=\left\langle p^{\lambda} G_{\sigma}, B_{0}\right\rangle / B_{0}$, then $a=y+$ $B_{0}$ with $y \in p^{\lambda} G_{\sigma}$ implies $y \in p^{\lambda} G_{\tau+1}$ if $\tau$ is sufficiently large (namely, if $y \in G_{\tau+1}$ and $\lambda(\tau)>\lambda$ ) because $B=p^{\lambda(\tau)} G_{\tau+1}$ and $G_{\tau+1} / B$ is a direct summand of $G_{\sigma} / B$. Since $\varepsilon_{\tau}$ preserves heights less than $\lambda(\tau)$ (computed in $C_{\tau}$ and $G_{\tau+1}$ ), there exists $x_{\tau}$ in $p^{\lambda} C_{\tau}$ such that $\varepsilon_{\tau}\left(x_{\tau}\right)=y$ and $\phi_{\tau}\left(x_{\tau}\right)=y+B_{0}=a$. Consequently, the mapping $\sum_{\tau<\sigma} \phi_{\tau}$ maps $p^{\lambda}\left(\sum_{\tau<\sigma} \oplus C_{\tau}\right)$ onto $p^{\lambda} A$ for each $\lambda<\mu$. This will prove to be an important feature in obtaining an almost balanced resolution of $A$.

We now construct another map from $C_{\tau}$ into $A$. This time we start with an epimorphism $p^{\lambda(\tau)} C_{\tau}[p] \rightarrow B[p]$. Since

$$
B=p^{\lambda(\tau+1)} G_{\tau+2} \subseteq p^{\lambda(\tau)+\omega} G_{\tau+2}=p^{\omega}\left(p^{\lambda(\tau)} G_{\tau+2}\right)
$$

and $p^{\lambda(\tau)} C_{\tau}$ is a direct sum of cyclic groups, the epimorphism $p^{\lambda(\tau)} C_{\tau}[p] \rightarrow B[p]$ can certainly be extended to a mapping (not necessarily epic) from $p^{\lambda(\tau)} C_{\tau}$ into $p^{\lambda(\tau)} G_{\tau+2}$. Since the extended map still does not decrease heights (computed in $C_{\tau}$ and $G_{\tau+2}$ ), it can be extended to a mapping from $C_{\tau}$ to $G_{\tau+2}$; let $\delta_{\tau}: C_{\tau} \rightarrow G_{\tau+2}$ with $\delta_{\tau}\left(p^{\lambda(\tau)} C_{\tau}[p]\right)=B[p]$. Denote by $\pi_{\tau}$ the composition of $\delta_{\tau}$ and the projection of $G_{\tau+2}$ onto $G_{\tau+2} / B_{0}$. Thus, we have $\pi_{\tau}: C_{\tau} \rightarrow G_{\tau+2} \rightarrow G_{\tau+2} / B_{0} \subseteq A$.

Some additional maps from totally projectives into $A$ are still required in order for us to obtain the desired resolution of $A$. Let $H_{\tau}$ be a $p^{\lambda(\tau)}$-high subgroup of $G_{\tau+1} / B_{0}$. Since $\lambda(\tau)$ is cofinal with $\omega$ and since $H_{\tau}$ is isotype and $p^{\lambda(\tau)}$-dense in $G_{\tau+1} / p^{\lambda(\tau)} G_{\tau+1}$, it is totally projective [18, Theorem A]; in fact, $H_{\tau} \cong G_{\tau+1} / p^{\lambda(\tau)} G_{\tau+1}$ (and therefore has $f_{\tau}^{\prime}$ for its Ulm-Kaplansky function). Let $i_{\tau}: H_{\tau} \subseteq G_{\tau+1} / B_{0} \subseteq A$ denote the inclusion map of $H_{\tau}$ into $A$. Define

$$
C=\sum_{\tau<\sigma} \oplus\left(C_{\tau} \oplus C_{\tau} \oplus H_{\tau}\right)
$$


and let $K$ be the kernel of the mapping $\sum_{\tau<\sigma}\left(\phi_{\tau}+\pi_{\tau}+i_{\tau}\right)$, which maps $C$ onto $A$. In fact, $\sum \phi_{\tau}$ is epic. We want to show that $(K, C)$ is an $A_{\mu}$-pair. Since $C_{\tau}$ and $H_{\tau}$ are totally projective so is $C$, and the length of $C$ is $\mu$. Therefore, to demonstrate that $(K, C)$ is an $A_{\mu}$-pair, it suffices to prove that $K$ is almost balanced in $C$ in the sense that

(i) $p^{\lambda} A=p^{\lambda}(C / K)=\left\langle p^{\lambda} C, K\right\rangle / K$ when $\lambda<\mu$, and

(ii) $K$ is isotype in $C$.

Condition (i) is a direct consequence of the fact that $\sum_{\tau<\sigma} \phi_{\tau}$ maps $p^{\lambda}\left(\sum_{\tau<\sigma} \oplus C_{\tau}\right)$ onto $p^{\lambda} A$ if $\lambda<\mu$. The proof that $K$ is isotype in $C$ is not quite as apparent. In order to show that $K$ is isotype in $C$, we use the following criterion. If $C$ is a $p$-group of limit length $\mu$ and $K$ is a subgroup of $G$, then $K$ is isotype in $G$ provided that

$$
p^{\lambda}(C / K)[p] \subseteq\left\langle p^{\lambda} C[p], K\right\rangle / K
$$

for each $\lambda<\mu$. It is easy to show by induction on $\alpha$ that this inclusion implies that $p^{\alpha} C \cap K \subseteq p^{\alpha} K$. Now, to show that condition (p) is, in fact, satisfied let $a \in p^{\lambda} A[p]$ $=p^{\lambda}(C / K)[p]$. From previous considerations we know that $a=y+B_{0}$, where $y \in p^{\lambda} G_{\tau+1}$ for a sufficiently large $\tau$. Since $H_{\tau}$ is $p^{\lambda(\tau)}$-high in $G_{\tau+1} / B_{0}$, it follows that

$$
\left(G_{\tau+1} / B_{0}\right)[p]=H_{\tau}[p]+p^{\lambda(\tau)}\left(G_{\tau+1} / B_{0}\right)[p] .
$$

Therefore, if $\lambda<\lambda(\tau)$, we can write

$$
y+B_{0}=h_{\tau}+\left(b+B_{0}\right),
$$

where $h_{\tau} \in p^{\lambda} H_{\tau}[p]$ and $b \in B=p^{\lambda(\tau)} G_{\tau+1}$. Since $p b \in B_{0}$ and $B_{0}$ is pure, we can choose $b \in B[p]$. Consequently, there exists $x \in p^{\lambda(\tau)} C_{\tau}[p]$ such that $\delta_{\tau}(x)=b$ and $\pi_{\tau}(x)=b+B_{0}$. Thus, we have produced an element $h_{\tau}+x$ in $p^{\lambda} C[p]$ that maps onto the given element $a$ in $A$ (under the mapping $\sum_{\tau<\sigma}\left(\phi_{\tau}+\pi_{\tau}+i_{\tau}\right)$ ). This completes the proof that $(K, C)$ is an $A_{\mu}$-pair.

Let $L$ be a totally projective group whose Ulm function is $f$ and set $H=K \oplus L$. Since $(H, C \oplus L)$ is an $A_{\mu}$-pair, $H$ is certainly an $A$-group. To complete the proof of the sufficiency of $(++)$, it remains only to show that $H$ has the desired invariants, namely,

$$
F_{0}(\alpha)=f(\alpha), \quad F_{\mu}(\alpha)=g(\alpha), \quad F_{\nu}(\alpha)=0 \quad \text { if } \nu \neq 0, \mu .
$$

The fact that $(H, C \oplus L)$ is an $A_{\mu}$-pair implies that $F_{\nu}(\alpha)=0$ unless $\nu$ is zero or $\mu$. Moreover, it is immediate from our construction that

$$
p^{\mu}((C \oplus L) / H)=p^{\mu}(C / K)=p^{\mu} A=B / B_{0}=E \text {. }
$$

Since the Ulm-Kaplansky function of $E$ is $g$, it must be that $F_{\mu}(\alpha)=g(\alpha)$. Finally, the $\alpha$ th Ulm-Kaplansky invariant of $C$ is either zero or else $f(\alpha)=m f(\alpha)$ due to the properties of $f_{\tau}$. Since $K$ is isotype in $C$, it quickly follows that $H=K \oplus L$ has $f$ for its Ulm-Kaplansky function since $L$ does. This completes the proof of the sufficiency of $(++)$.

Necessity. Suppose now that $H$ is an $A$-group. Then, by definition, $H=\sum_{i \in I} \oplus H_{i}$, where $\left(H_{i}, G_{i}\right)$ is an $A_{\mu(i)}$-pair for distinct limit ordinals $\mu(i)$ not cofinal with $\omega$. As usual, let the $A$-invariants of $H$ be denoted by $F_{0}(\alpha)$ and $F_{\mu}(\alpha)$, where $\mu \in \mathscr{M}$ $=\{\mu(i): i \in I\}$. 
Set $f(\alpha)=F_{0}(\alpha)$ and $g_{\mu}(\alpha)=F_{\mu}(\alpha), \mu \in \mathscr{M}$. We need to show that the condition $(++)$

$$
\int_{\lambda}^{\lambda+\omega} f(\alpha) \geqslant \sum_{\lambda<\mu} \int_{0}^{\infty+} g_{\mu}(\alpha)
$$

is satisfied. Since the $A$-invariants are additive, it suffices to establish $(++)$ for a $\mu$-elementary $A$-group $H$. However, in this case $(++)$ simplifies and takes the form

$$
\int_{\lambda}^{\lambda+\omega} f(\alpha) \geqslant \int_{0}^{\infty+} g_{\mu}(\alpha)
$$

for $\lambda<\mu$, where $(H, G)$ is an $A_{\mu}$-pair. Furthermore, it is enough to show that $\int_{\lambda}^{\mu} f(\alpha) \geqslant \int_{0}^{\infty+} g_{\mu}(\alpha)$ is satisfied because $f$ is admissible, since it is the Ulm-Kaplansky function of $H$. Recall that $g_{\mu}$ is the extended Ulm-Kaplansky function of $E_{\mu}=p^{\mu}(G / H)$. Let $p^{\alpha} H[p]=S_{\alpha} \oplus p^{\alpha+1} H[p]$ and set $S=\sum_{\lambda \leqslant \alpha<\mu} \oplus S_{\alpha \cdot}$. Choose $T$ so that $S \subseteq T \subseteq p^{\lambda} G[p]$ and $\langle T, H\rangle / H=E_{\mu}[p]$; this is possible since $H$ is isotype in $G$ and since $E_{\mu}=p^{\mu}(G / H)=\bigcap_{\lambda<\mu}\left\langle p^{\lambda} G, H\right\rangle / H$. Consider $S$ and $T$ as valuated vector spaces (whose values are heights computed in $G$ ) and observe that $S$ is dense in $T$, that is, if $t \neq 0$ belongs to $T$ then $|t-s|>|t|$ for some $s \in S$. However, since $G$ is totally projective the valuated vector space $G[p]$ is contained in a free space $F$. Therefore, we conclude that $T$ is no larger (in cardinality) than $S$ since no subspace of a free space can have a smaller dense subspace. Hence,

$$
\int_{\lambda}^{\mu} f(\alpha)=|S|=|T| \geqslant\left|E_{\mu}[p]\right| \geqslant \int_{0}^{\infty+} g_{\mu}(\alpha)
$$

for each $\lambda<\mu$, and the theorem is proved.

REMARK. Since it is obviously necessary for the functions $f=F_{0}$ and $g_{\mu}=F_{\mu}$ to be admissible in order to qualify as the $A$-invariants for an $A$-group $H$, an equivalent form of the existence theorem is the following. Let $f$ and $g_{\mu}, \mu \in \mathscr{M}$, be ordinal-tocardinal functions, where $\mathscr{M}$ is a collection of limit ordinals not cofinal with $\omega$. A necessary and sufficient condition for the existence of an $A$-group with $A$-invariants

$$
F_{0}(\alpha)=f(\alpha), \quad F_{\mu}(\alpha)=g_{\mu}(\alpha) \quad \text { if } \mu \in \mathscr{M}, F_{\nu}(\alpha)=0 \quad \text { if } \nu \neq 0 \text { and } \nu \notin \mathscr{M},
$$

is that $f$ and $g_{\mu}$, for each $\mu \in \mathscr{M}$, be admissible and $(++)$ be satisfied.

By combining the existence and uniqueness theorems, we obtain a complete classification of $A$-groups.

THEOREM 5. The class of A-groups are in 1-1 correspondence, via their A-invariants, with the class of sequences of admissible functions $\left\{F_{\mu}\right\}, \mu=0$ or a limit ordinal not cofinal with $\omega$, that satisfy condition $(++)$ upon setting $f=F_{0}$ and $g_{\mu}=F_{\mu}$ if $\mu \neq 0$. The given correspondence preserves finite and infinite sums.

6. The structure and properties of $A$-groups. In this section we formulate some of the most important properties of $A$-groups. In cases where these results are immediate consequences of the complete classification of $A$-groups provided by Theorem 5, proofs are either omitted entirely or only short proofs are included. However, because of their significance to the theory, we label these results (including direct corollaries) as theorems.

Our first result shows how to identify among $A$-groups the well-known special types simply by looking at the invariants. 
THeOREM 6. Let $H$ be an $A$-group with $A$-invariants $F_{\mu}(\alpha), \mu \geqslant 0$. Then $H$ is totally projective if and only if $F_{\mu}(\alpha)=0$ when $\mu>0$ for all $\alpha$ (including $\alpha=\infty$ ). Moreover, $H$ is an $S$-group if and only if $F_{\mu}(\alpha)=0$ when $\mu>0$ for all $\alpha$ different from $\infty$. Finally, $H$ is an $N$-group if and only if $F_{\mu}(\alpha)=0$ unless $\mu=0$ or $\mu=\omega_{1}$ and $\alpha=0$.

The next result implies, among other things, that every $A$-group $H$ has a totally projective summand $T$ such that all the isotype subgroups of $H$ containing $T$ (including $T$ and $H$ ) have exactly the same Ulm-Kaplansky invariants.

THEOREM 7. If $H$ is an A-group, then $H=T \oplus K$, where $K$ is an A-group and $T$ is totally projective and has the same Ulm-Kaplansky invariants as $H$.

Proof. Apply Lemma D and Theorem 5.

Recall that an $A$-group $H$ is a $\mu$-elementary $A$-group if $F_{\nu}(\alpha)=0$, for every $\alpha$, unless $\nu=0$ or $\nu=\mu$. If, in addition, $F_{\mu}(\infty)=0$, we say that $H$ is an adjusted $\mu$-elementary $A$-group.

THEOREM 8. Any A-group $H$ can be written as $H=T \oplus S \oplus A$, where $T$ is totally projective and where $S=\Sigma \oplus S_{\mu}$ and $A=\Sigma \oplus A_{\mu}$ are direct sums of $\mu$-elementary $S$-groups and adjusted $\mu$-elementary A-groups, respectively.

Proof. Let $H$ be an $A$-group with $A$-invariants $F_{\mu}(\alpha), \mu=0$ or a limit ordinal not cofinal with $\omega$. It is understood that $\mu$ ranges over the appropriate ordinals. Set $f(\alpha)=F_{0}(\alpha)$ and $g_{\mu}(\alpha)=F_{\mu}(\alpha)$ if $\mu \neq 0$. By Lemma D we can decompose the function $f$ as $f=f+\sum_{\mu \neq 0} f_{\mu}$ so that $f_{\mu}$ is admissible of length $\mu$ and totally dominates $g_{\mu}$ in the sense that $\int_{\lambda}^{\mu} f_{\mu}(\alpha) \geqslant \int_{0}^{\infty+} g_{\mu}(\alpha)$ when $\lambda<\mu$. Clearly, we can further decompose the function $f_{\mu}$ as $f_{\mu}=f_{S, \mu}+f_{A, \mu}$ into admissible functions $f_{S, \mu}$ and $f_{A, \mu}$ so that, when $\lambda<\mu$,

$$
\int_{\lambda}^{\mu} f_{S, \mu}(\alpha) \geqslant g_{\mu}(\infty) \text { and } \int_{\lambda}^{\mu} f_{A, \mu}(\alpha) \geqslant \int_{0}^{\infty} g_{\mu}(\alpha)
$$

According to the existence theorem, there are $A$-groups $T, S_{\mu}$, and $A_{\mu}$ that have the following prescribed $A$-invariants:

$$
\begin{gathered}
T: F_{0}(\alpha)=f(\alpha) \quad\left(F_{\mu}(\alpha)=0 \text { if } \mu \neq 0\right), \\
S_{\mu}: F_{0}(\alpha)=f_{S, \mu}(\alpha) ; \quad F_{\mu}(\alpha)= \begin{cases}0 & \text { if } \alpha \neq \infty \\
g_{\mu}(\infty) & \text { if } \alpha=\infty\end{cases} \\
\left(F_{\nu}(\alpha)=0 \text { if } \nu \neq 0 \text { and } \nu \neq \mu\right), \\
A_{\mu}: F_{0}(\alpha)=f_{A, \mu}(\alpha) ; \quad F_{\mu}(\alpha)= \begin{cases}g_{\mu}(\alpha) & \text { if } \alpha \neq \infty \\
0 & \text { if } \alpha=\infty\end{cases} \\
\quad\left(F_{\nu}(\alpha)=0 \text { if } \nu \neq 0 \text { and } \nu \neq \mu\right) .
\end{gathered}
$$

Obviously, $A_{\mu}$ is an adjusted $\mu$-elementary $A$-group, while $S_{\mu}$ can be decomposed into $\mu$-elementary $S$-groups (with $\mu$-fixed) if it is not already. Since the $A$-group $T \oplus$ $\sum_{\mu \neq 0} S_{\mu} \oplus \sum_{\mu \neq 0} \oplus A_{\mu}$ has the same $A$-invariants as $H$, the theorem follows.

It perhaps should be noted in passing that $H$ is an adjusted $\mu$-elementary $A$-group if and only if $H$ is an isotype subgroup of a totally projective group $G$ of length $\mu$ 
such that $G / H$ is a reduced totally projective and $p^{\lambda}(G / H)=\left\langle p^{\lambda} G, H\right\rangle / H$ when $\lambda<\mu$. In particular, an $N$-group is always an adjusted $\mu$-elementary $A$-group with $\mu=\omega_{1}$.

The next theorem generalizes the same result for totally projective groups (Nunke [13]) and $S$-groups (Warfield [18]).

THEOREM 9. Let $H$ be an arbitrary reduced p-group and $\alpha$ an arbitrary ordinal. The $H$ is an $A$-group if and only if both $p^{\alpha} H$ and $H / p^{\alpha} H$ are $A$-groups.

Proof. The "only if" part is easy. First, it reduces quickly to the case where $H$ is a $\mu$-elementary $A$-group. Thus suppose that $(H, G)$ is an $A_{\mu}$-pair for some limit ordinal $\mu$ not cofinal with $\omega$. If $\alpha<\mu$, it is routine to verify that $\left(p^{\alpha} H, p^{\alpha} G\right)$ is an $A_{\lambda}$-pair, where $\mu=\alpha+\lambda$. Thus $p^{\alpha} H$ is an $A$-group since $p^{\alpha} H=0$ if $\alpha \geqslant \mu$. Likewise, if $\alpha<\mu,\left\langle H, p^{\alpha} G\right\rangle / p^{\alpha} G$ is balanced in $G / p^{\alpha} G$ and the quotient is totally projective. Hence, $H / p^{\alpha} H=\left\langle H, p^{\alpha} G\right\rangle / p^{\alpha} G$ is totally projective if $\alpha<\mu$, and in any event $H / p^{\alpha} H$ is an $A$-group.

To prove the converse, we first observe that $H$ is an $A$-group if $p^{n} H$ is for a positive integer $n$. Suppose that $p^{n} H$ is an $A$-group. Let $p^{n} H=\sum \oplus K_{i}$, where $K_{i}$ is a $\mu(i)$-elementary $A$-group for each $i$. We can lift the decomposition of $p^{n} H=$ $\sum \oplus K_{i}$ to a decomposition of $H$ (see, for example, Theorem 11 in [8]). Therefore, let $H=\Sigma \oplus H_{i}$, where $p^{n} H_{i}=K_{i}$. If $\left(K_{i}, T_{i}\right)$ is an $A_{\mu(i)}$-pair, then $\left(H_{i}, G_{i}\right)$ is also an $A_{\mu(i)}$-pair for a suitable totally projective group $G_{i}$ with $p^{n} G_{i}=T_{i}$. Hence, $H=$ $\sum \oplus H_{i}$ must be an $A$-group if $p^{n} H$ is an $A$-group.

Now, suppose $\alpha$ is arbitrary and both $p^{\alpha} H$ and $H / p^{\alpha} H$ are $A$-groups. Let $\alpha=\beta+n$, where $\beta$ is a limit and $n<\omega$. From what has preceded, we conclude that $p^{\beta} H$ and $H / p^{\beta} H$ are $A$-groups. Our objective, of course, is to prove that $H$ is an $A$-group, and we shall do this based on the fact that $p^{\beta} H$ and $H / p^{\beta} H$ are for a limit ordinal $\beta$. We may assume without loss of generality that $p^{\beta} H \neq 0$. It is rather immediate that $H$ is at least an isotype subgroup of a totally projective group, for if $p^{\beta} H$ is isotype in $p^{\beta} G$ the identity map on $p^{\beta} H$ can be extended to a mapping from $H$ to $G$ since $H / p^{\beta} H$ is weakly $p^{\beta}$-projective [7, Theorem 2.2]. Under our hypothesis we may assume that $G$, as well as $p^{\beta} G$, is totally projective. Clearly, if we combine such a map from $H$ to $G$ with $H \rightarrow H / p^{\beta} H \nrightarrow G^{\prime}$, we can obtain the desired embedding of $H$ in a totally projective group $G \oplus G^{\prime}$. A benefit of this is that we now know that the Ulm-Kaplansky function of $H$ is admissible [18, Theorem 4.7].

Letting $f_{G}$ denote the Ulm-Kaplansky function of an arbitrary group $G$ and letting $p^{\beta} H=H(\beta)$, we define the function $f_{H(\beta)}^{\prime}$ by the rule

$$
f_{H(\beta)}^{\prime}(\alpha)= \begin{cases}0 & \text { if } \alpha<\beta, \\ f_{H(\beta)}(\gamma) & \text { if } \alpha=\beta+\gamma .\end{cases}
$$

Note that $f_{H(\beta)}^{\prime}$ is not itself an Ulm-Kaplansky function, but rather a shift of the Ulm-Kaplansky function $f_{H(\beta)}$. Since $\beta$ is a limit, $f_{H}=f_{H / p^{\beta} H}+f_{p^{\beta} H}^{\prime}$. Denote the nonzero $A$-invariants of $H / p^{\beta} H$ as follows:

$$
F_{0}=f_{H / p^{\beta} H}, \quad F_{\mu}=g_{\mu} \quad \text { for } 0 \neq \mu \in \mathscr{M} .
$$


Observe that no $\mu \in \mathscr{M}$ can exceed $\beta$ (since $H / p^{\beta} H$ has length $\beta$ ). Likewise, denote the nonzero $A$-invariants of $H(\beta)=p^{\beta} H$ as follows:

$$
F_{0}=f_{H(\beta)}, \quad F_{\mu}=g_{\beta+\mu} \quad \text { for } \beta<\beta+\mu \in \mathscr{M}^{\prime} .
$$

Note that $\mathscr{M}$ and $\mathscr{M}^{\prime}$ are disjoint. Hereafter in this proof it is understood that $\mu$ ranges over $\mathscr{M} \cup \mathscr{M}^{\prime}$. If $\lambda \leqslant \beta$,

$$
\int_{\lambda}^{\lambda+\omega} f_{H}(\alpha) \geqslant \int_{\beta}^{\infty} f_{H}(\alpha)=\int_{0}^{\infty} f_{H(\beta)}(\alpha)
$$

since $f_{H}$ is admissible. Therefore, if $\lambda$ is less than some $\mu$,

$$
\int_{\lambda}^{\lambda+\omega} f_{H}(\alpha) \geqslant \int_{\lambda}^{\lambda+\omega} f_{H / p^{\beta} H}(\alpha)+\int_{0}^{\infty} f_{H(\beta)}(\alpha) \geqslant \sum_{\mu>\lambda} \int_{0}^{\infty+} g_{\mu}(\alpha) .
$$

Consequently, there exists an $A$-group $K$ whose $A$-invariants are closely related to $H$, namely,

$$
F_{0}^{K}=f_{H}, \quad F_{\mu}^{K}=g_{\mu} \text { if } \mu \neq 0
$$

Recall that

$$
g_{\mu}(\alpha)= \begin{cases}F_{\mu}^{H / p^{\beta} H}(\alpha) & \text { if } \mu \in \mathscr{M}, \\ F_{\nu}^{p^{\beta} H}(\alpha) & \text { if } \mu=\beta+\nu \in \mathscr{M}^{\prime} .\end{cases}
$$

Thus, $p^{\beta} K$ and $p^{\beta} H$ are $A$-groups with the same invariants, and therefore $p^{\beta} K \cong p^{\beta} H$. Likewise, $K / p^{\beta} K \cong H / p^{\beta} H$. Finally, $K \cong H$ by virtue of a recent result of Hill and Megibben [9] that implies that an $A$-group is totally Zippin, which means (by definition) that an isomorphism $p^{\beta} H \nrightarrow p^{\beta} K$ can be extended to an isomorphism $H \nrightarrow K$ provided the quotients $H / p^{\beta} H$ and $K / p^{\beta} K$ are isomorphic and totally Zippin. Thus, $H$ is an $A$-group since $K$ is an $A$-group and the theorem is proved.

The existence theorem is evidence enough that $A$-groups exist in abundance. However, we include the following examples to illustrate how naturally they occur.

EXAMPLE 1 . let $\Omega=\omega_{1}$ and let $G$ be a totally projective group of length $\Omega 2$. Let $M$ be Megibben's example in [11, p. 109] identified in the d.s.c. group $p^{\Omega} G$. If $H$ is maximal in $G$ with respect to $H \cap p^{\Omega} G=M$, then $H$ is an $A$-group that is not an $S$-group. Note that $H / p^{\Omega} H$ is an $S$-group.

EXAMPLE 2 . Let $W$ be an $S$-group of length $\Omega$ that is not totally projective and let $T$ be totally projective of length greater than $\Omega$. If $W$ is isotype and $p^{\Omega}$-dense in the d.s.c. group $G$, then the $p^{\Omega}$-pure exact sequence $\operatorname{TOR}(W, T) \leadsto \operatorname{TOR}(G, T) \rightarrow \sum \oplus$ $T$ reveals that $\operatorname{TOR}(W, T)$ is an $A$-group (but not an $S$-group).

The following result is particularly striking since it was only in 1964 that a subgroup of a d.s.c. was first exhibited [12] that did not itself decompose into a d.s.c. Shortly after, it was shown that no isotype subgroup of a d.s.c. of countable length could fail to decompose into countable groups. Moreover, all isotype subgroups of d.s.c.'s that are $S$-groups must decompose into groups of cardinality at most $\aleph_{1}$.

THEOREM 10. There exist arbitrarily large A-groups that are, in fact, isotype subgroups of d.s.c.'s that cannot be written as the direct sum of smaller groups. (Hence they are quasi-indecomposable.) 
Proof. Let $\boldsymbol{\aleph} \geqslant \boldsymbol{\aleph}_{1}$ be a cardinal and let $\sigma$ be the first ordinal of cardinality $\boldsymbol{\aleph}$. Set $\mu=\omega_{1}$ and define

$$
f(\alpha)= \begin{cases}\aleph & \text { if } \alpha<\mu \\ 0 & \text { otherwise (if } \alpha \text { is uncountable) }\end{cases}
$$

Define

$$
g_{\mu}(\alpha)= \begin{cases}\boldsymbol{\kappa} & \text { if } \alpha \leqslant \sigma \\ 0 & \text { otherwise (if } \alpha \text { exceeds } \sigma)\end{cases}
$$

Note that $g_{\mu}$ has length $\sigma+1$, not $\sigma$. Obviously, $f$ and $g_{\mu}$ are admissible and $f$ totally dominates $g_{\mu}$ since

$$
\int_{\lambda}^{\mu} f(\alpha)=\aleph=\int_{0}^{\infty+} g_{\mu}(\alpha)
$$

Therefore, there is a $\mu$-elementary $A$-group $H$ that has for its $A$-invariants $F_{0}(\alpha)=$ $f(\alpha)$ and $F_{\mu}(\alpha)=g_{\mu}(\alpha)$. Since $\mu=\omega_{1}$, there is a d.s.c. group $G$ such that $(H, G)$ is an $A_{\mu}$-pair. The proof of the existence theorem reveals that $H$ has cardinality $\aleph$. To demonstrate that $H$ cannot be decomposed as a direct sum of smaller groups, assume that it can. Suppose that $H=\Sigma \oplus H_{i}$, where $\left|H_{i}\right|<\boldsymbol{N}$ for each $i$. Consequently, $g_{\mu}$ must decompose as $g_{\mu}=\sum g_{\mu, i}$, where $g_{\mu, i}$ is admissible and $\int_{0}^{\infty} g_{\mu, i}(\alpha) \leqslant$ $\left|H_{i}\right|<\aleph$. But this is absurd, since for at least one $i$ we must have $g_{\mu, i}(\sigma) \neq 0$, which implies that $\int_{0}^{\infty} g_{\mu, i}(\alpha) \geqslant \aleph$ since $g_{\mu, i}$ is admissible.

Our final result shows that there is no class of reduced $p$-groups that is closed with respect to direct sums properly containing the class of $A$-groups whose members are determined by their $A$-invariants. Here, in the more general setting, we define $E_{\mu}$ $=\overline{\left(H / p^{\mu} H\right)} /\left(H / p^{\mu} H\right)$.

THEOREM 11. Let $\mathscr{C}$ be a class of reduced p-groups closed with respect to direct sums (and such that membership is independent of notation). Suppose that the A-invariants determine the structure of all the members of $\mathscr{C}$. If $\mathscr{C}$ contains the A-groups then it is exactly the class of $A$-groups.

Proof. Suppose that $E$ belongs to $\mathscr{C}$. Since $E \oplus H$ and $H$ have the same $A$-invariants for some $A$-group $H$ and since both belong to $\mathscr{C}$, it is evident that $E \oplus H=H$ and $E$ is a direct summand of an $A$-group. Thus we know already that the only members of $\mathscr{C}$ that are not $A$-groups, if any, must be summands of $A$-groups. We shall demonstrate, however, that summands of $A$-groups belonging to $\mathscr{C}$ are, in fact, $A$-groups. This will be accomplished if we can show that a summand of an $A$-group has the same invariants as some $A$-group.

Let $H$ be an $A$-group and let $H=K \oplus L$. We prove that the $A$-invariants of $K$ agree with the $A$-invariants of some $A$-group by induction on the length $\sigma$ of $K$. This is certainly the case if $\sigma$ is countable, so assume that $\sigma$ is uncountable. By employing Theorem 9, we may assume that $\sigma=\beta+\sigma$ for each limit ordinal $\beta<\sigma$. For otherwise $p^{\beta} K$ and $K / p^{\beta} K$ have the same invariants as $A$-groups by the induction hypothesis, and therefore so does $K$. Observe that $E_{\mu}(H)=E_{\mu}(K) \oplus E_{\mu}(L)$ and, of course, $f_{H}=f_{K}+f_{L}$. Since $f_{K}$ is admissible and $\int_{\beta}^{\beta+\omega} f_{K}(\alpha) \geqslant \int_{\gamma}^{\gamma+\omega} f_{K}(\alpha)$ when 
$\beta<\gamma$, we may assume without loss of generality (for some fixed cardinal $m$ ) that

$$
\int_{\beta}^{\beta+\omega} f_{K}(\alpha)=m=\int_{\gamma}^{\gamma+\omega} f_{K}(\alpha)
$$

whenever $\beta$ and $\gamma$ are limit ordinals less that $\sigma$. Again, since $f_{K}$ is admissible, it is clear that $m \geqslant|\sigma|$. By the same argument used in the proof of the necessity of condition $(++)$ in the proof of the existence theorem, we can obtain the inequality

$$
\int_{\lambda}^{\mu} f_{K}(\alpha) \geqslant\left|E_{\mu}(K)[p]\right|
$$

for each $\lambda<\mu$. Therefore, if $\lambda<\mu$,

$$
m \geqslant\left|E_{\mu}(K)[p]\right| \geqslant \int_{0}^{\infty+} g_{\mu}(\alpha)
$$

where $g_{\mu}=F_{\mu}{ }^{K}$ is the extended Ulm-Kaplansky function of $E_{\mu}(K)$. Since $g_{\mu}$ does not exist (or is zero) when $\mu>\sigma$, and since $m \geqslant|\sigma|$, the preceding inequality yields

$(++) \quad \int_{\lambda}^{\lambda+\omega} f_{K}(\alpha) \geqslant m \geqslant \sum_{\lambda<\mu} \int_{0}^{\infty+} g_{\mu}(\alpha)$.

Therefore, according to the existing theorem, $K$ has the same $A$-invariants as some $A$-group, and the theorem is proved.

There remains at least one significant open question about $A$-groups. Are they closed with respect to direct summands? We suspect (and there is some historical basis for this opinion) that the summand question is relatively difficult. At least its solution has not yet fallen to our particular attack.

\section{REFERENCES}

1. P. Crawley and A. Hales, The structure of abelian p-groups given by certain presentations, J. Algebra 12 (1969), 10-23.

2. L. Fuchs, Infinite abelian groups, Vol. 2, Academic Press, New York, 1973.

3. P. Hill, Isotype subgroups of direct sums of countable groups, Illinois J. Math. 13 (1969), 281-290.

4. $\quad$ The third axiom of countability for abelian groups, Proc. Amer. Math. Soc. 82 (1981), $347-350$.

5. On the classification of abelian groups, photocopied manuscript, 1967.

6. On the classification of $N$-groups, Houston J. Math. 10 (1984), 43-55.

7. P. Hill and C. Megibben, On direct sums of countable groups and generalizations, Studies on Abelian Groups, Dunod, Paris, 1968, pp. 183-206.

8. Extending isomorphisms and lifting decompositions on abelian groups, Math. Ann. 175 (1968), 159-168.

9. On the theory and classification of abelian p-groups, Math. Z. (to appear).

10. G. Kolettis, Direct sums of countable groups, Duke Math. J. 27 (1960), 111-125.

11. C. Megibben, On $p^{\alpha}$-high injectives, Math. Z. 122 (1971), 104-110.

12. R. Nunke, On the structure of Tor, Proc. Colloq. Abelian Groups, Budapest, 1964, pp. 115-124.

13. , Homology and direct sums of countable abelian groups, Math. Z. 101 (1967), 182-212.

14. R. Hunter, F. Richman and E. Walker, Warfield modules, Lecture Notes in Math., vol. 616, Springer-Verlag, New York, 1977, pp. 57-74.

15. L. Parker and E. Walker, An extension of the Ulm-Kolettis theorems, Studies on Abelian Groups, Dunod, Paris, 1968, pp. 309-325.

16. H. Ulm, Zur Theorie der abzahlbar-unendlichen abelschen Gruppen, Math. Ann. 107 (1933), 774-803. 
17. K. Wallace, On mixed groups of torsion-free rank one with totally projective primary components, J. Algebra 17 (1971), 482-488.

18. R. Warfield, A classification theorem for abelian p-groups, Trans. Amer. Math. Soc. 210 (1975), 149-168.

19. The structure of mixed abelian groups, Lecture Notes in Math., vol. 616, Springer-Verlag, 1977, pp. 1-38.

20. L. Zippin, Countable torsion groups, Ann. of Math. (2) 36 (1935), 86-99.

Department of Mathematics, Auburn University, Auburn, Alabama 36849 